\title{
Reducing flow-induced noise and vibrations by resonant metamaterials with multiple stop band behavior
}

\author{
Felipe Alves Pires* and Hervé Denayer ${ }^{\dagger}$ \\ KU Leuven, Leuven, Belgium \\ DMMS Lab, Flanders Make, Belgium \\ Elke Deckers $\ddagger$ \\ $K U$ Leuven, Diepenbeek, Belgium \\ DMMS Lab, Flanders Make, Belgium \\ Claus Claeys ${ }^{\S}$, Wim Desmet ${ }^{\text {I }}$ \\ KU Leuven, Leuven, Belgium \\ DMMS Lab, Flanders Make, Belgium
}

\begin{abstract}
In the search for novel Noise, Vibrations and Harshness (NVH) solutions, vibro-acoustic locally resonant metamaterials (LRMs) have recently emerged, combining a good NVH behavior and a lightweight design in desired frequency regions, referred to as stop bands i.e. frequency zones of strong vibration and/or noise attenuation. This paper experimentally investigates the potential of using LRMs with multiple stop band behavior to reduce flow-induced noise and vibrations of coupled vibro-acoustic systems. In a first stage, the vibrations of a flat plate excited by a turbulent flow under the influence of vortex-shedding are analyzed and a metamaterial solution for a multiple stop band behavior is designed. In a second stage, a hard-walled backing-cavity is attached to the system and the noise radiation due to the plate's vibrations into the backing cavity is assessed. The flat plate is then treated with the designed resonant additions and the results show that the metamaterial solution is able to suppress the consequential noise radiation to the coupled cavity due to flow-induced vibrations phenomena.
\end{abstract}

\section{Introduction}

Flow-induced induced cabin noise has become a major topic for a variety of high speed vehicles. In the automotive sector, for example, the vibrations that occur on the underbody of a moving car are generated by an unsteady turbulent flow, which cause noise to be radiated into the car's cabin. Comparatively, in an aircraft, the pressure fluctuations of the turbulent flow developed over the aircraft's fuselage can lead to high levels of noise inside the cabin, directly affecting the comfort of passengers [1].

A variety of such NVH phenomena are related to a turbulent flow around bluff bodies, for which different crosssectional areas are considered [2]. For instance, for a bluff rod with a circular shape, as a result of boundary layer roll-up, alternating vortices in a Karman-vortex-street pattern are formed and shed, which continue to grow under continuous injection of circulation from the cylinder shear layers until they become strong enough to draw the shear layers across the wake [3]. These vortices stop building up in strength and shed away from the cylinder as the vorticity with an opposite sign in sufficient concentration cuts further supply of circulation to the forming vortex [4]. This phenomenon is often referred to as vortex-shedding $[2,5]$.

Different techniques to mitigate flow-induced noise and vibrations have been studied and developed. These solutions may include the addition of mass and changes in the system's stiffness [6,7]. The use of damping layers [8] as well as active control approaches [9] have also been studied. In the study of [6], for example, passive techniques, such as the addition of mass, damping and skin stiffness are investigated and applied to an aircraft fuselage. In [9], an active control approach is applied to reduce the flow-induced noise transmitted through a plate. However, a great number of

\footnotetext{
*PhD Researcher, Department of Mechanical Engineering, Division LMSD, Celestijnenlaan 300-box 2420, 3001 Leuven, Belgium.

${ }^{\dagger}$ Senior Researcher, Department of Mechanical Engineering, Division LMSD, Celestijnenlaan 300-box 2420, 3001 Leuven, Belgium.

$¥$ Assistant Professor, Diepenbeek Campus, Mechanical Engineering Technology TC, Wetenschapspark 27, 3590 Diepenbeek, Belgium.

${ }^{\S}$ Senior Researcher, Department of Mechanical Engineering, Division LMSD, Celestijnenlaan 300-box 2420, 3001 Leuven, Belgium.

"TFull Professor, Department of Mechanical Engineering, Division LMSD, Celestijnenlaan 300-box 2420, 3001 Leuven, Belgium.
} 
these solutions are rather heavy and bulky and/or may not perform desirably in the lower frequency range [10]. For that reason, novel compact and lightweight countermeasures are needed to tackle the challenges of not only achieving a good $\mathrm{NVH}$ performance but also ensuring a lightweight design.

Locally resonant metamaterials have recently emerged and proven to hold potential to combine a lightweight design and good NVH performance in desired frequency regions, typically referred to as stop bands (SB). These are frequency zones with no free wave propagation [11] and can be created by introducing resonant additions on a subwavelength scale [12] to a host structure, due to a Fano-type interference [13, 14]. These zones of strong wave attenuation can be predicted by means of dispersion diagrams. These can be calculated by a unit cell (UC) modeling approach, by applying Bloch-Floquet periodicity boundary conditions $[15,16]$ in the finite element (FE) model of a single representative UC.

In fact, the potential of LRMs was investigated previously for a turbulent flow excitation in $[17,18]$. In the former, a metamaterial solution was developed to tackle TBL-induced noise and vibrations of a cavity-backed flat plate. The applied solution has shown to not only reduce the vibrations of the flat plate but also the consequential noise radiation into the backing cavity. In the latter, a metamaterial solution was applied targeting a tonal vibration amplification region caused by vortex-shedding. However, the study did not consider the noise radiated due to these vortex-induced vibrations. Indeed, as shown in both studies, there are two possible causes of tonal issues: (i) the periodic flow features causing a tonal excitation due to vortex-shedding and dynamics of the system i.e. vibro-acoustic modes. This paper presents an approach to tackle both issues simultaneously. It proposes the use of LRMs with multiple stop band behavior to reduce flow-induced noise and vibrations caused by a vortex street in the wake of a cylindrical rod and it is an extension of the aforementioned works. This study considers the same hard-walled backing-cavity used in [17], which will be attached to the flat plate excited by vortex-shedding in [18]. The vibrations of the flat plate as well as the noise radiation into the cavity are experimentally analyzed. Two realizable metamaterial designs are proposed to treat two frequency ranges of the system in study. The experiments are repeated and the metamaterial performance to improve the vibro-acoustic responses of the systems is evaluated.

The paper is organized as follows. Section II introduces the host structure as well as the metamaterial designs to achieve multiple stop band behavior used in the investigation. Section III shows the experimental results of the flow-induced vibrations of the metamaterial plate. Lastly, Section IV presents a summary of tht main findings of the paper.

\section{Problem definition}

This section presents an evaluation of the pressure fluctuations of the considered excitation as well as the experimental configuration of the host structure and the metamaterial design used to obtain stop band behavior.

\section{A. Flow configurations}

Normally, the flow-induced NVH behavior can be generated by different aerodynamic loads, thus, studying the potential of LRMs to target different flow phenomena is important. In fact, two flow-induced phenomena are considered in this work: (i) turbulent boundary layer and (ii) vortex-shedding induced noise and vibrations of a cavity-backed flat plate. In the former, a grazing flow with speed $U=19 \mathrm{~m} / \mathrm{s}$ is considered and here used as reference. This corresponds to a Reynolds number $\operatorname{Re}=2.610^{6}$ and Mach number $\mathrm{M}=0.056$. In the latter, the same flow speed but with a bluff cylinder of diameter $\mathrm{D}_{c}=6 \mathrm{~mm}$ fixed upstream to create vortex-shedding is considered.

The cylinder is positioned at a distance close enough to the top duct wall so that the turbulent wake can reach it. A ratio $\mathrm{h} / \mathrm{D}_{c} \approx 1.0$ i.e. relationship between the distance of the bluff cylinder with respect to the top surface and the bluff body's diameter, is utilized, as shown in Figure 1. The vibro-acoustic system in study is placed where the top wall is indicated in the figure. In addition, an array of microphones is also used in the investigation such that it is possible to learn about the flow characteristics for the considered aerodynamic loads. Such array is placed in the same location as the vibro-acoustic system. This is allowed since the test set-up used in the study enables that different features can be easily interchanged while maintaining their position. Information about the characteristics of the vibro-acoustic system as well as the flow condition is given in the next sections. 

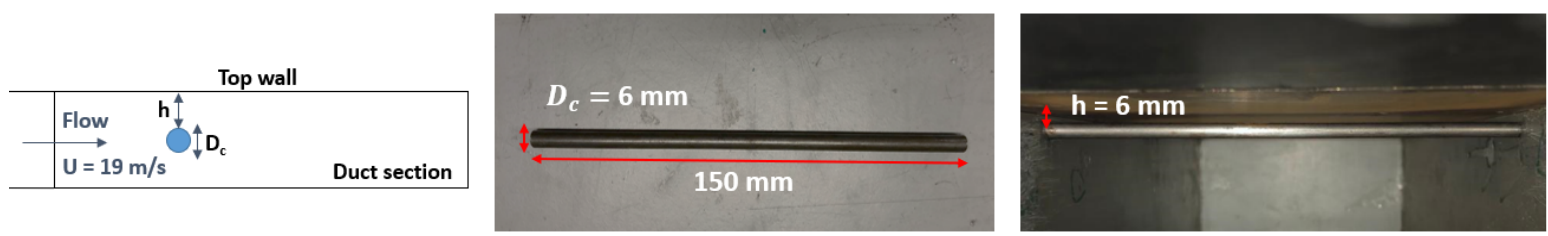

Fig. 1 Illustration of a realized bluff cylinder and how it is placed inside the duct section.

\section{B. Pressure fluctuations assessment}

In fact, when vortex-shedding is studied, the vortex-shedding frequency $\mathrm{f}_{s}=\mathrm{St} \mathrm{U} / \mathrm{D}_{c}$ can be predicted, where $\mathrm{U}$, St, $v$ and $\mathrm{D}_{c}$ are the free stream velocity, the Strouhal number, the fluid kinematic viscosity and the bluff cylinder diameter. Considering a St $\approx 0.20$ [19], a $\mathrm{f}_{s} \approx 630 \mathrm{~Hz}$ is predicted. However, given the relatively high Reynolds number for the conditions in study, it is possible that a regular von Karman street can no longer exist and a broadband pressure spectrum can be achieved [20], where the highest levels are expected nevertheless around $\mathrm{St} \approx 0.2$.

In order to have an indication of the characteristics of the aerodynamic loads that this work uses as excitation of the system, the pressure fluctuations for each flow condition are measured. This is performed by an array of 30 flush-mounted $1 / 4$ " microphones, in a $5 \times 6$ grid, on a side-wall of a duct section of a small wind tunnel with cross-sectional dimensions $150 \times 75 \mathrm{~mm}$, where the host structure in study is placed in a later stage, sufficiently distant from a rootsblower, as shown in Figure 2 b).

a)

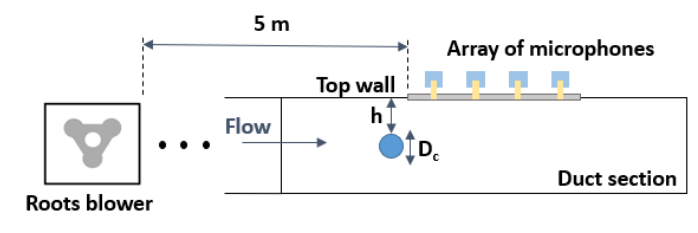

b)

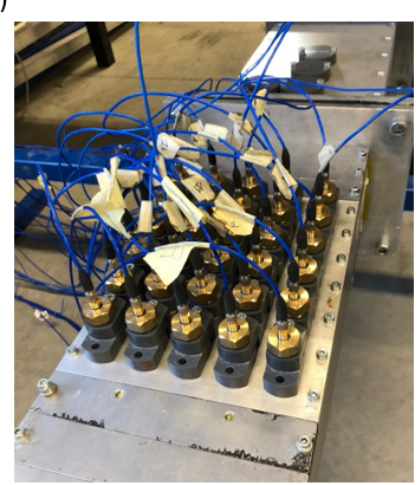

Fig. 2 a) Schematic and b) Realized overview of the experimental setup to measure pressure fluctuations.

Figure 3 illustrates the autopower spectra of the pressure fluctuations for the grazing flow and vortex-shedding cases. As can be seen, the addition of the bluff cylinder upstream leads to higher pressure levels in which two peaks are present: (i) one near the predicted vortex-shedding frequency and (ii) another at a higher frequency. The two vortex-shedding peaks may occur due to the fact that the flow velocity inside the duct is not uniform, since the flow is highly turbulent for the given Reynolds number. 


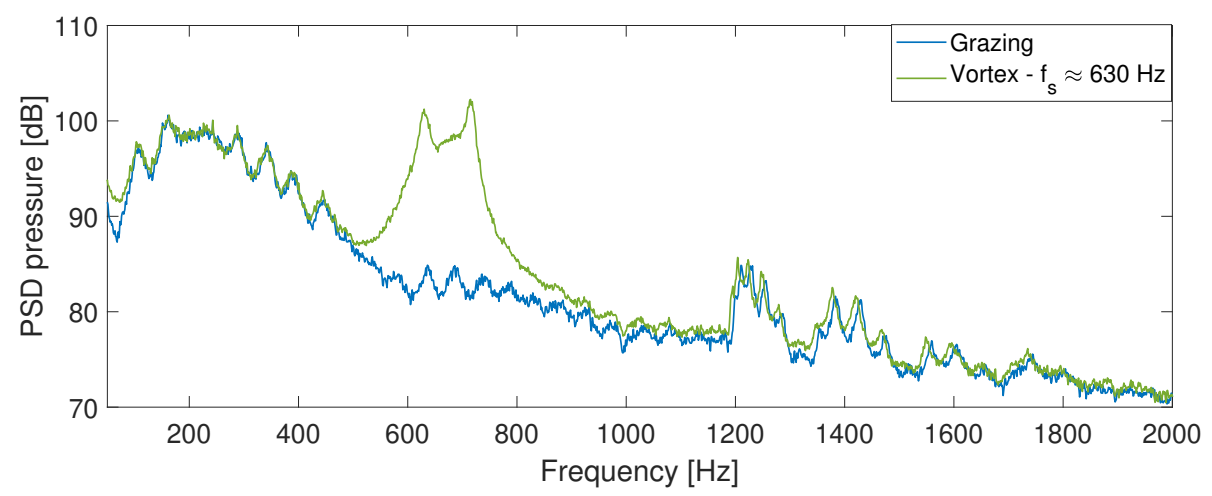

Fig. 3 Autopower pressure comparison between the grazing and vortex-shedding pressure fluctuations cases for $\mathbf{U}=19 \mathrm{~m} / \mathrm{s}$. Reference pressure - $20 \mu \mathrm{Pa}$.

\section{Host Structure and acoustic cavity}

With the goal of assessing the use of LRMs to reduce flow-induced noise and vibrations, a steel flat plate with dimensions $150 \times 200 \times 0.5 \mathrm{~mm}$ is used as a host structure and is attached to the duct section where the pressure fluctuations were measured with the array of microphones for the considered flow cases. Such combination between material and dimensions is chosen such that a flexible structure with pronounced modal behavior in the low-frequency range below $1000 \mathrm{~Hz}$ is obtained. The steel host structure has density $\rho=7766.9 \mathrm{~kg} / \mathrm{m}^{3}$, Young's Modulus E $=233.1 \mathrm{GPa}$, Poisson's Ratio $v=0.27$ and Structural damping $=0.2 \%$. The flat plate is clamped and mounted flush as a side wall of a duct section, as shown in Figure 4. A backing cavity made of aluminium with two microphones is mounted over the flexible plate to study the noise radiation due to the flow-induced loads. Bolts are used to attached the cavity to the system.

a)

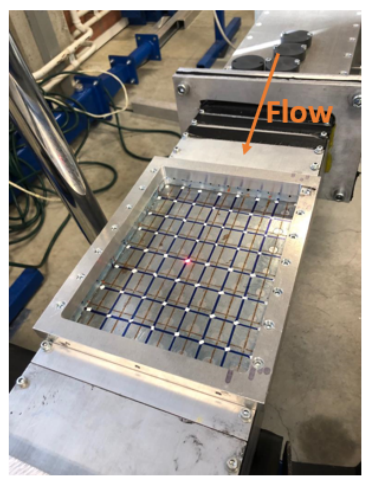

b)

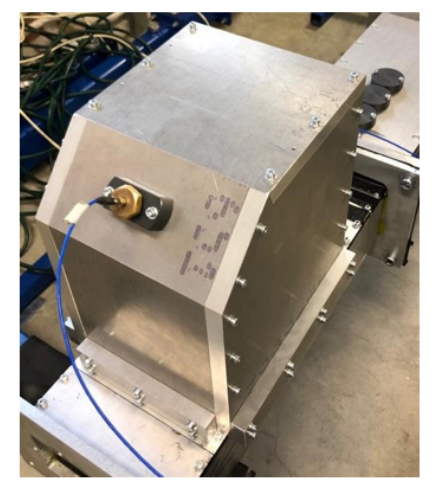

Fig. 4 Illustration of the a) steel flat plate and b) backing cavity attached to the system [18].

\section{Unit cell modeling and metamaterial design}

Since LRMs typically have a periodic arrangement, the unit cell modelling approach can be used. This modelling strategy allows to obtain the wave propagation in an infinite periodic structure based on a model of one unit cell of the structure and the application of the Bloch-Floquet periodic BCs. In this paper the FEM framework is used and an undamped structure is considered such that stop bands can be predicted based on the evaluation of the irreducible brillouin contour in the wave domain. For more details on this modelling strategy, the interested reader is referred to $[11,16,21,22]$.

As studied in [23], different resonator designs can be combined in a single structure to create multiple stop bands. The present work utilizes this premise to obtain a combination of stop band behavior for the flat plate under the 
considered turbulent flow excitation for two phenomena: (i) one resonator design to suppress the forced vibrations of a flat plate under a vortex-shedding excitation and the consequential noise radiation into a backing cavity and (ii) another metamaterial design to reduce the noise radiation due to the excitation of a vibro-acoustic mode of the system which is at a different frequency than the vortex-shedding frequency exciting the plate. With this approach, it is possible to verify if a metamaterial solution can tackle various flow-induced $\mathrm{NVH}$ problems simultaneously by creating two stop band effect regions.

In order to design a metamaterial solution, the dynamic behavior of the structure of interest needs to be evaluated. In this case, the vibrational response of the flat plate in Figure 4 with no flow is known from [18]. The authors reported that the considered structure has a pronounced dynamic behavior below $1000 \mathrm{~Hz}$ with the highest peak amplitude around $600 \mathrm{~Hz}$ for the considered conditions. For that reason, a metamaterial solution to tackle this vortex-shedding frequency region is designed, which is here referred to as design $\mathbf{A}$ and shown in Figure 5 a). The second metamaterial solution is designed to target the first acoustic mode of the cavity attached to the system, which is at $724 \mathrm{~Hz}$, as depicted in Figure 5 b), is here referred to as design $\mathbf{B}$. The two resonator designs possess a similar mass, namely $0.59 \mathrm{~g}$ for design $\mathbf{A}$ and $0.55 \mathrm{~g}$ for design $\mathbf{B}$. They correspond to $29 \%$ and $27 \%$ of mass addition w.r.t. the mass of a single unit cell for designs $\mathbf{A}$ and $\mathbf{B}$, respectively. The resonators are made of the same material i.e. polymethyl methacrylate (PMMA), whose material properties are shown in Table 1.

To be able to compare, in a later stage, the metamaterial reduction performance with an equivalent mass case, a block with no resonant behavior within the targeted frequency range and with similar added mass as the resonators, namely, $0.57 \mathrm{~g}$, is also designed, as illustrated in Figure $5 \mathrm{c}$ ). This mass value corresponds to $28 \%$ of mass addition w.r.t. the mass of one unit cell. The difference in mass values between the realized resonant and non-resonant additions is due to manufacturing imprecision and it should not affect the conclusions significantly.

a)

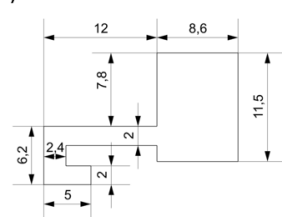

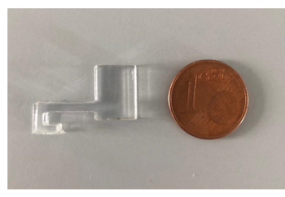

c)

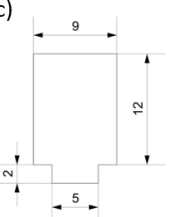

b)

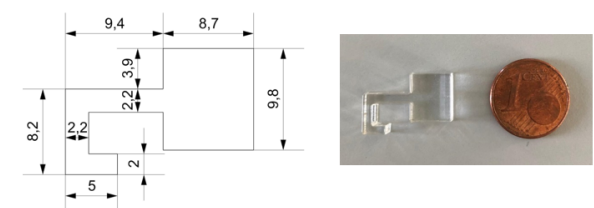

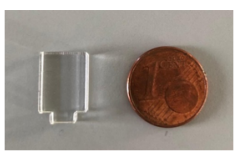

Fig. 5 Illustration of the PMMA a) resonator design $A$ b) resonator design $B$ and c) non-resonant structure design. A realized example of each structure is also shown. All structures are $4 \mathbf{m m}$ thick.

Table 1 Material properties of PMMA [23].

\begin{tabular}{cccc}
\hline Young's Modulus & Density & Poisson's Ratio & Structural Damping \\
\hline $4850 \mathrm{MPa}$ & $1188.38 \mathrm{~kg} / \mathrm{m}^{3}$ & 0.31 & $5 \%$ \\
\hline
\end{tabular}

\section{E. Metamaterial configurations}

To achieve stop band behavior, the resonant structures are added and glued to the steel plate by Loctite ${ }^{\circledR} 406$ contact adhesive in a rectangular pattern with a spacing of $21 \times 25 \mathrm{~mm}$, which ensures the subwavelength spacing between the resonant additions needed to target the bending waves of interest. A total of 56 resonators can be added to the steel host structure. Four cases are considered in this work:

- Bare case: flat plate without resonant structures, used as a reference.

- Single resonator type (SRT) case: flat plate entirely covered with 56 added resonators of design A, which target flexural waves propagating around $600 \mathrm{~Hz}$, as in Figure 6 a). In this case, the metamaterial solution adds in total $29 \%$ of mass w.r.t. the mass of the bare plate. 
- Mixed case: flat plate with 28 resonators of design A alternated with 28 resonators of design $\mathbf{B}$ mixed, as shown in Figure 6 b). The total mass addition for this metamaterial configuration corresponds to approximately $28 \%$ w.r.t. the mass of the bare plate.

- Equivalent mass case: flat plate entirely covered with non-resonant additions. For this case, the total mass addition is $28 \%$ w.r.t. the mass of the bare plate.

It is worth pointing out that the case which the plate is entirely covered with resonators of design $\mathbf{B}$ is not considered here since the main goal of the paper is to target a frequency range where vortex-shedding is expected [18] and to check if by combing resonators tuned at different frequencies can also lead to noise radiation reduction at multiple frequency regions.

a)

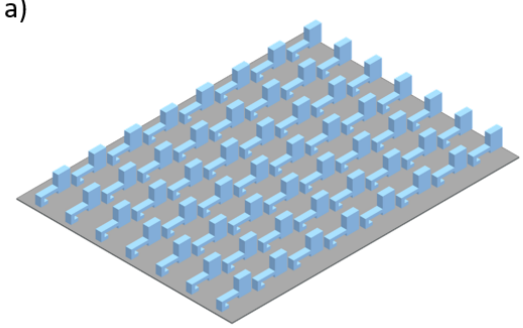

b)

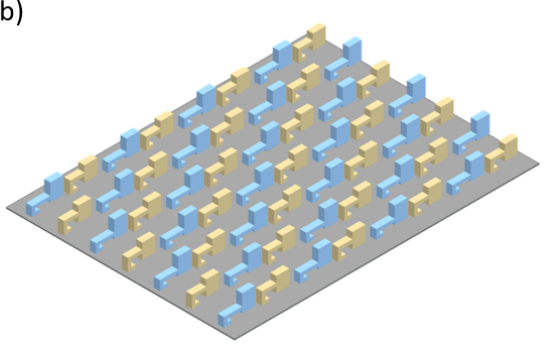

Fig. 6 Visualization of the considered configurations a) SRT case b) mixed case.

\section{F. Stop band prediction}

The predicted stop bands of the metamaterial designs are illustrated in Figure 7. The different curves in the dispersion diagrams are distinguished according to the ratio of out-of-plane versus in-plane motion of the host structure. This analysis shows that a stop band for flexural waves, identified by their out-of-plane motion, is created from $583.2 \mathrm{~Hz}$ to $634.9 \mathrm{~Hz}$ for the SRT case. For the mixed case, two stop bands are predicted: one stop band opens from $566.8 \mathrm{~Hz}$ to $605 \mathrm{~Hz}$ which is related to the resonators design $\mathbf{A}$ and another created from $736.3 \mathrm{~Hz}$ to $800.2 \mathrm{~Hz}$, which is related to resonators design $\mathbf{B}$. These stop bands are smaller than for the SRT case and for when the plate is fully treated with resonators of design $\mathbf{B}$ [17] and this is due to the fact that only half of the resonators are tuned to the same natural frequency within a same area. In addition, they also lie in a slightly lower frequency range, and this is due to the interaction of the two different tuned locally resonant additions [24].

a)

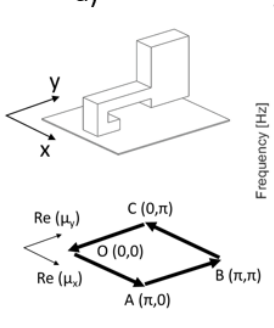

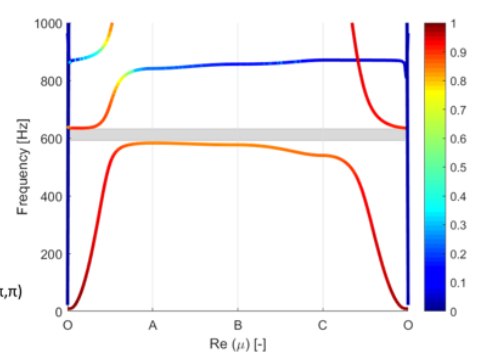

b)

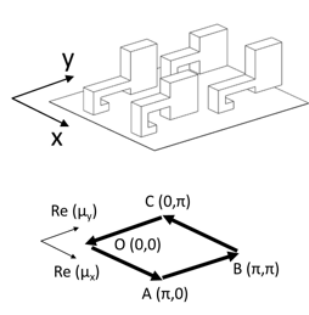

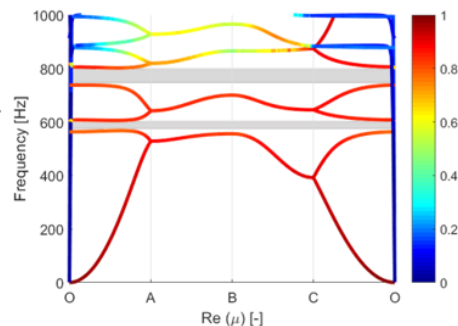

Fig. 7 Representation of the a) IBC with a sketch of the respective wave space and dispersion diagram for the SRT case and b) IBC with a sketch of the respective wave space and dispersion diagram for the mixed case. The gray shaded area represents the predicted stop bands for the two configurations. The color bar represents the ratio according to the out-of-plane and in-plane motion of the waves, where 0 is total in-plane and 1 total out-of-plane.

\section{Experimental results - tackling tonal issues simultaneously}

This section discusses the results for the experimentally measured vibrations of the flat plate excited by the wake of a bluff object. The vibrations of the flat plate are experimentally analyzed. The metamaterial design to create multiple 
stop band is then applied. The experiments are repeated and the metamaterial performance to improve the vibro-acoustic responses of the system is evaluated.

\section{A. Vortex-induced vibrations of the flat plate}

The effect of vortex shedding in the wake of a nearby bluff obstacle on the flow-induced vibrations of the plate has been studied in [18]. The structure illustrated in Figure 4 a) is excited by turbulent fluctuating pressures due to a turbulent grazing flow of air that runs inside the considered small wind tunnel at a speed $\mathrm{U}=19 \mathrm{~m} / \mathrm{s}$. The following results are a summary of what has been discussed in the aforementioned study.

The vibrations of the bare flat plate due to the turbulent flow are measured at 72 points by Scanning Laser Doppler Vibrometer (SLDV) and the results are presented as the RMS over all points of the power spectral density spectra of velocity. These results are used as a reference for comparison with the vortex-shedding case. Subsequently, the flat plate is then excited by a turbulent flow for when a solid bluff rod of diameter $\mathrm{D}_{c}=6 \mathrm{~mm}$ is fixed upstream to create a vortex-shedding for the considered mean flow velocity of $U=19 \mathrm{~m} / \mathrm{s}$, as investigated in II.B. Figure 8 shows the obtained comparison between the vibrational response spectra of the bare plate excited by a grazing turbulent flow in the presence of vortex-shedding. As addressed in [18], the addition of the rod leads to a significant amplification of the mode of the plate. around the frequency range where higher pressures are expected with the addition of the bluff object upstream, addressed in Section II.B.

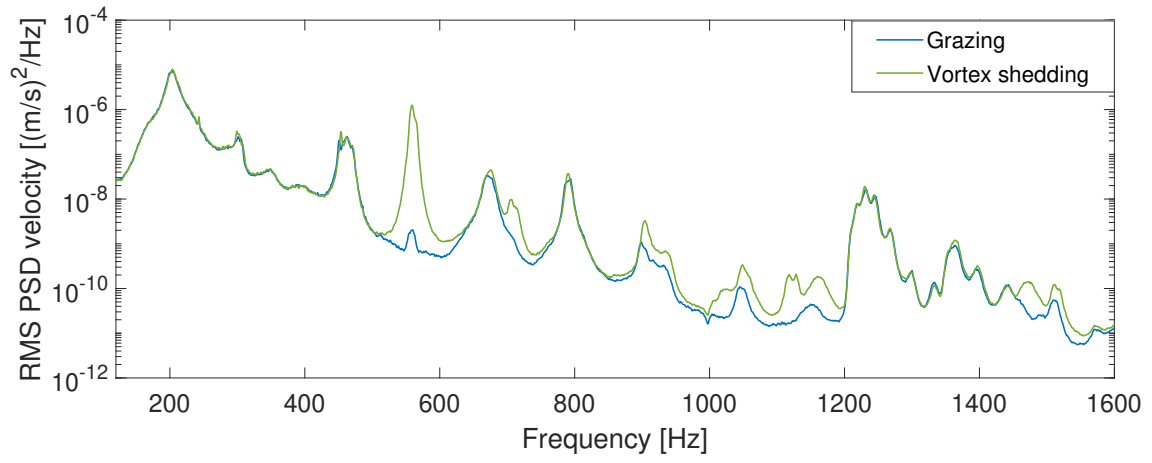

Fig. 8 Comparison of RMS PSD velocity response of the bare plate for a grazing flow and a flow with a cylinder fixed upstream causing vortex-shedding.

The flat plate is then treated with the considered configurations, addressed in Section II.E. Figure 9 illustrates the responses for the bare and the metamaterial configurations when the cylinder is placed upstream to cause vortex-shedding. For the SRT configuration, i.e. when the entire plate is treated with resonators design A, a strong dip can be observed on the spectrum around the predicted stop band, which limits are indicated by the orange solid vertical lines. For the mixed configuration, where the structure is treated with two types of resonators, two dips are found on the spectrum: (i) one around $600 \mathrm{~Hz}$ related to the added resonators design $\mathbf{A}$ and (ii) another dip at a higher frequency, namely, around $750 \mathrm{~Hz}$, related to the added resonators design $\mathbf{B}$. In the former, the dip is less pronounced then for the case when the entire plate is treated since, in this case, less resonators targeting the bending waves around $600 \mathrm{~Hz}$ are added. The stop band limits for the mixed case are indicated with the yellow dash-dotted vertical lines. 


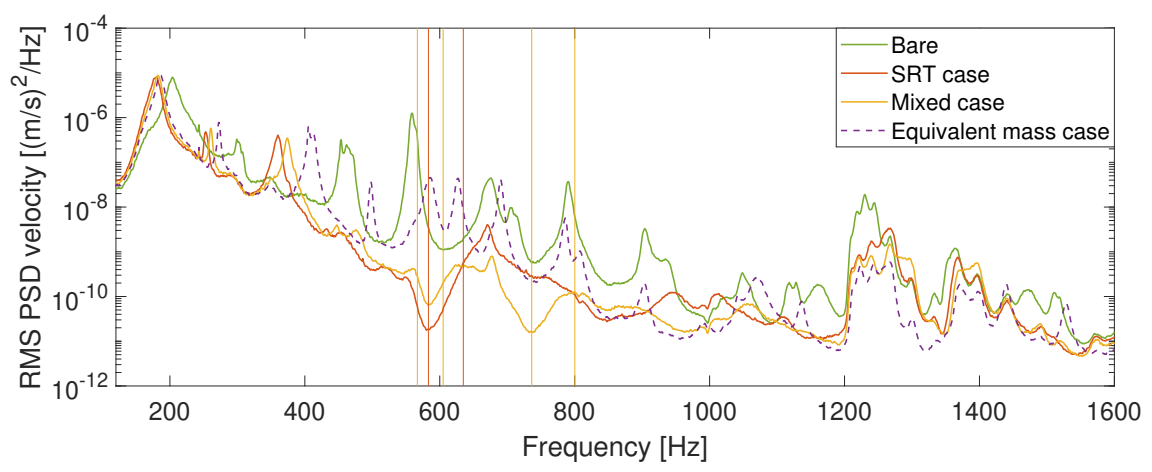

Fig. 9 Experimental RMS PSD velocity response of the flat plate for the considered configurations with a cylinder fixed upstream to create vortex-shedding.

\section{B. Vortex-induced noise radiation into a cavity-backed plate}

In order to investigate the use of LRMs to mitigate the noise radiated due to the vortex-induced vibrations of the flat plate, this work uses the cavity-backed plate setup, shown in Figure 4 b). An evaluation of the sound pressure levels (SPL) inside the system is carried out for the flow cases, considering again the grazing flow excitation as a reference. Figure 10 illustrates the comparison of SPL inside the bare cavity-backed plate under grazing flow and vortex-shedding excitation for $\mathrm{U}=19 \mathrm{~m} / \mathrm{s}$. The results indicate that the amplified structural mode leads to a higher overall SPL inside the cavity-backed plate around the frequency range of $600 \mathrm{~Hz}$.

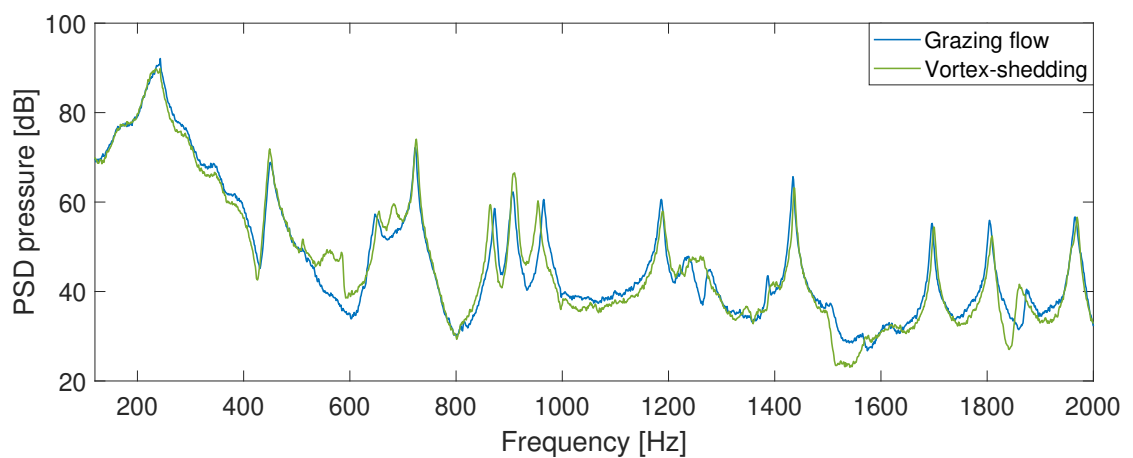

Fig. 10 Comparison of PSD pressure of the bare cavity-backed plate under a grazing flow and flow with a cylinder fixed upstream to create vortex-shedding, measured on the microphone shown in Figure 4 b). Reference pressure - $20 \mu \mathrm{Pa}$.

An analysis of the noise radiation into the backing cavity is also performed for the considered metamaterial configurations, as shown in Figure 11. The vertical lines represent the predicted stop bands, illustrated similarly as in previous section. Two affected frequency regions are noticed: (i) the region where vortex-shedding occurs and (ii) the region of the first vibro-acoustic mode of the cavity-backed plate. In the former, a $13 \mathrm{~dB}$ reduction is achieved in the noise radiated due to the amplified mode for the mixed case, which is $5 \mathrm{~dB}$ less than the SRT case, whose reason is explained earlier regarding the number of added resonators targeting the bending waves around $600 \mathrm{~Hz}$. In the latter, a $9 \mathrm{~dB}$ reduction is obtained in the first acoustic driven mode of the system at $724 \mathrm{~Hz}$ for the mixed case, which is an additional $5 \mathrm{~dB}$ reduction when compared with the SRT configuration, for which a small reduction was obtained, possibly due to the damping in the resonators. Besides, the mixed metamaterial configuration also outperforms the equivalent case. The fact that the amplitude for the equivalent mass case is slightly higher than the bare case at $724 \mathrm{~Hz}$ might be due to experimental error, as no significant effect of mass addition is expected at acoustic driven modes. However, this will be further investigated. These results are an indication that a combined pattern of resonators is eligible to tackle flow-induced NVH issues of systems, where multiple frequency ranges need to be considered. 


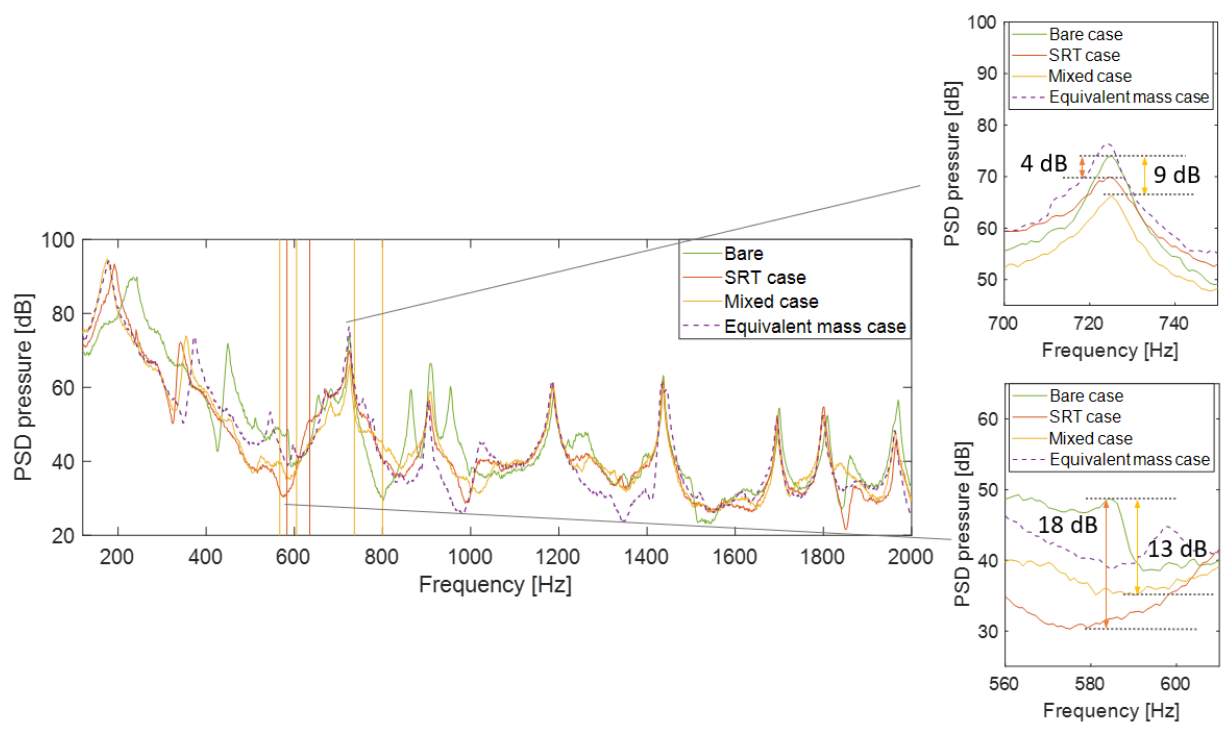

Fig. 11 Comparison of PSD pressure response of the cavity-backed plate with and without metamaterials for when a cylinder is fixed upstream to create vortex-shedding, measured on the microphone shown in Figure 4 b). Reference pressure - $20 \mu \mathrm{Pa}$.

\section{Conclusions}

This paper presented an experimental investigation on the potential of using LRMs to suppress flow-induced noise and vibrations. Two different flow conditions are used, namely, a grazing flow, which is used as reference, and a flow under the presence of vortex-shedding. To achieve this goal, a vibro-acoustic system comprised by a cavity-backed plate is used.

This work presented the use of LRMs with multiple stop band behavior to tackle two issues simultaneously of the cavity-backed plate system under vortex-shedding excitation: (i) dynamics of the system i.e. vibro-acoustic modes and (ii) the periodic flow features due to vortex-shedding. For the latter, it was verified that the addition of the bluff component led to an amplification of a structural mode, which is due to the zone of increased pressure levels with respect to a grazing flow.

The proposed mixed LRM configuration led to two vibration reduction zones, though less pronounced than the case in which the entire plate is treated since less resonators targeting the bending waves around the frequency range of interest were added. With regard to noise radiation, two frequency regions were affected: (i) the region where vortex-shedding is expected and (ii) the region of the first acoustic-driven mode of the cavity-backed plate. The designed metamaterial solution with multiple stop band behavior was able to not only mitigate the noise radiated due to the amplified mode but also reduce the pressure levels of the first acoustic driven mode of the vibro-acoustic system. The proposed solution also outperformed the equivalent mass case.

\section{Acknowledgments}

The research of F. A. Pires is funded by an Early Stage Researcher grant within the European Project SMARTANSWER Marie Curie Initial Training Network (GA 722401). This research was partially supported by Flanders Make, the strategic research centre for the manufacturing industry. The Research Fund KU Leuven is gratefully acknowledged for its support.

\section{References}

[1] Maury, C., Gardonio, P., and Elliott, S., "A wavenumber approach to modelling the response of a randomly excited panel, Part II: Application to aircraft panels excited by a turbulent boundary layer," Journal of Sound and Vibration, Vol. 252, No. 1, 2002, pp. 115-139. 
[2] Nakamura, T., Kaneko, S., Inada, F., Kato, M., Ishihara, K., Nishihara, T., Mureithi, N. W., and Langthjem, M. A., Flow-induced vibrations: classifications and lessons from practical experiences, Butterworth-Heinemann, 2013.

[3] Gerrard, J., "The mechanics of the formation region of vortices behind bluff bodies," Journal of fluid mechanics, Vol. 25, No. 2, 1966, pp. 401-413.

[4] Lau, Y., So, R., and Leung, R., "Flow-induced vibration of elastic slender structures in a cylinder wake," Journal of fluids and structures, Vol. 19, No. 8, 2004, pp. 1061-1083.

[5] Zdravkovich, M. M., Flow around circular cylinders: Volume 2: Applications, Vol. 2, Oxford university press, 1997.

[6] Barton, C., and Mixson, J., "Noise transmission and control for a light twin-engine aircraft," Journal of aircraft, Vol. 18, No. 7, 1981, pp. 570-575.

[7] Graham, W., "Boundary layer induced noise in aircraft, Part I: The flat plate model," Journal of Sound and Vibration, Vol. 192, No. 1, 1996, pp. 101-120.

[8] Park, J., Mongeau, L., and Siegmund, T., "An investigation of the flow-induced sound and vibration of viscoelastically supported rectangular plates: experiments and model verification," Journal of sound and vibration, Vol. 275, No. 1-2, 2004, pp. $249-265$.

[9] Maury, C., Gardonio, P., and Elliott, S. J., "Active control of the flow-induced noise transmitted through a panel," AIAA journal, Vol. 39, No. 10, 2001, pp. 1860-1867.

[10] Camussi, R., Noise sources in turbulent shear flows: fundamentals and applications, Vol. 545, Springer Science \& Business Media, 2013.

[11] Brillouin, L., Wave propagation in periodic structures: electric filters and crystal lattices, Courier Corporation, 2003.

[12] Liu, Z., Zhang, X., Mao, Y., Zhu, Y., Yang, Z., Chan, C. T., and Sheng, P., "Locally resonant sonic materials," science, Vol. 289, No. 5485, 2000, pp. 1734-1736.

[13] Goffaux, C., Sánchez-Dehesa, J., Yeyati, A. L., Lambin, P., Khelif, A., Vasseur, J., and Djafari-Rouhani, B., "Evidence of Fano-like interference phenomena in locally resonant materials," Physical review letters, Vol. 88, No. 22, 2002, p. 225502.

[14] Celli, P., and Gonella, S., "Heterogeneity meets disorder: anomalous wave transport in telescopic metamaterials," arXiv preprint arXiv:1703.08522, 2017.

[15] Mead, D., "Wave propagation in continuous periodic structures: research contributions from Southampton, 1964-1995," Journal of sound and vibration, Vol. 190, No. 3, 1996, pp. 495-524.

[16] Hussein, M. I., "Reduced Bloch mode expansion for periodic media band structure calculations," Proceedings of the Royal Society A: Mathematical, Physical and Engineering Sciences, Vol. 465, No. 2109, 2009, pp. 2825-2848.

[17] Alves Pires, F., Claeys, C., Sangiuliano, L., Denayer, H., Deckers, E., and Desmet, W., "Suppression of flow-induced noise and vibrations by locally resonant metamaterials," AIAA AVIATION 2020 FORUM, 2020, p. 2586.

[18] Alves Pires, F., Denayer, H., Deckers, E., Claeys, C., and Desmet, W., "Reduction of vortex-induced vibrations by locally resonant metamaterials," PROCEEDINGS OF ISMA2020 AND USD2020, 2020.

[19] Shih, W., Wang, C., Coles, D., and Roshko, A., "Experiments on flow past rough circular cylinders at large Reynolds numbers," Journal of Wind Engineering and Industrial Aerodynamics, Vol. 49, No. 1-3, 1993, pp. 351-368.

[20] Belloli, M., Giappino, S., Morganti, S., Muggiasca, S., and Zasso, A., "Vortex induced vibrations at high Reynolds numbers on circular cylinders," Ocean Engineering, Vol. 94, 2015, pp. 140-154.

[21] Claeys, C. C., Vergote, K., Sas, P., and Desmet, W., "On the potential of tuned resonators to obtain low-frequency vibrational stop bands in periodic panels," Journal of Sound and Vibration, Vol. 332, No. 6, 2013, pp. 1418-1436.

[22] Bloch, F., "Über die quantenmechanik der elektronen in kristallgittern,” Zeitschrift für physik, Vol. 52, No. 7-8, 1929, pp. 555-600.

[23] Claeys, C., de Melo Filho, N. G. R., Van Belle, L., Deckers, E., and Desmet, W., "Design and validation of metamaterials for multiple structural stop bands in waveguides," Extreme Mechanics Letters, Vol. 12, 2017, pp. 7-22.

[24] de Melo Filho, N., Claeys, C., Deckers, E., and Desmet, W., "Realisation of a thermoformed vibro-acoustic metamaterial for increased STL in acoustic resonance driven environments," Applied Acoustics, Vol. 156, 2019, pp. 78-82. 